\title{
GENERALIZATIONS OF THE VITALI-HAHN-SAKS THEOREM ON VECTOR MEASURES
}

\author{
RICHARD A. OBERLE
}

\begin{abstract}
In this paper an extension of the Brook's control measure existence theorem for families of vector measures is established. This result is applied to pointwise convergent sequences of finitely additive vector measures to obtain a generalization of the Vitali-Hahn-Saks theorem.
\end{abstract}

Introduction. The results presented in this paper began with an attempt to extend the classical Vitali-Hahn-Saks theorem to sequences of finitely additive vector measures. The work of Bogdanowicz [8], Drewnowski [5], and Labuda [6] indicated that the Baire category argument could be used to establish a vector form of the Vitali-Hahn-Saks theorem for sequences convergent on a delta ring (as opposed to a $\sigma$-algebra) provided the delta ring was endowed with an appropriate sequentially complete topology. Earlier, Ando [1], using a lemma due to Phillips [9] as a substitute for the Baire category argument, noted the validity of the Vitali-Hahn-Saks theorem for sequences of finitely additive scalar measures on a $\sigma$-algebra. Later, Brooks and Jewett [4] generalized Phillips' lemma to vector functions and obtained a Vitali-Hahn-Saks theorem for sequences of strongly bounded vector measures convergent pointwise on a $\sigma$-algebra. Combining the methodology developed by Ando with the Brooks-Jewett formulation of Phillips' lemma yields a formulation of the Vitali-Hahn-Saks theorem which pulls together the ideas developed in the above listed extensions. The results also lead to a more refined knowledge of the control measure existence theorems developed by Bartle, Dunford, and Schwartz [2], Brooks [3], and Drewnowski [5].

Let $V$ denote a ring of subsets of an abstract space $X$, let $N$ denote the positive integers and let $\left(R^{+}\right) R$ denote the (nonnegative) reals. Denote by $C(V)$ the space of all subadditive and increasing functions, from the ring $V$ into $R^{+}$, which are zero at the empty set. The space $C(V)$ is called the space of contents on the ring $V$ and elements are referred to as contents. A sequence of sets $A_{n} \in V, n \in N$, is said to be dominated if there exists a set $B \in V$ such that $A_{n} \subseteq B$ for $n=1,2,3, \ldots$ A content $p \in C(V)$ is said to be Rickart on the ring $V$ if $\lim _{n} p\left(A_{n}\right)=0$ for each dominated, disjoint sequence $A_{n} \in V, n \in N$. A set of contents $P \subset C(V)$ is said to be uniformly Rickart on the ring $V$ if the above limit holds uniformly with

Received by the editors August 11, 1976.

AMS (MOS) subject classifications (1970). Primary 28A45, 28A10, 28 A60.

C American Mathematical Society 1978 
respect to the contents $p \in P$. This condition is an abstraction of the condition of strong boundedness introduced by Rickart [11].

A content $p \in C(V)$ is said to be upper complete if each sequence $A_{n} \in V$, $n \in N$, for which $\Sigma_{n} p\left(A_{n}\right)<\infty$, is dominated. Any nonnegative measure on a $\sigma$-ring restricted to the delta ring of sets of finite measure is an upper complete content.

The ring $V$ is an abelian group with respect to the symmetric difference operation $\div$ and each content $p \in C(V)$ generates a semimetric on the group $(V, \div)$ by the relation $\rho(A, B)=p(A \div B)$ for sets $A, B \in V$. This semimetric is invariant in the sense that $\rho(A \div C, B \div C)=\rho(A, B)$ for sets $A, B, C \in V$. Therefore, any family of contents $P \subset C(V)$ generates a topology on the group $(V, \div)$. A pair $(V, P)$, where $P \subset C(V)$ and $(V, \div)$ is given the topology generated by the family $P$, will be called a topological ring of sets. Characterizations of sequential completeness for a topological ring may be found in [6] and [8]. Two families of contents $P$ and $Q$ are said to be equivalent if each $q \in Q$ is $P$-continuous and conversely. That is, equivalent families of contents generate equivalent topologies. A content $p \in$ $C(V)$ is said to control a family $Q \subset C(V)$ if the family $Q$ is $p$-equicontinuous.

Let $Y$ denote a real Banach space and let $N$ denote the positive integers. For a ring of sets $V$, denote by $a(V, Y)$, respectively $c a(V, Y)$, the space of $Y$-valued, finitely additive (countably additive) vector functions on $V$. Elements of this space are called vector charges (volumes). For each vector charge $\mu \in a(V, Y)$, the semivariation $p(\cdot, \mu): V \rightarrow[0, \infty]$ is defined on a set $A \in V$ by the relation

$$
p(A, \mu)=\sup (|\mu(B)|: B \in V, B \subseteq A) .
$$

Denote by $a b(V, Y)$ the family of all charges $\mu \in a(V, Y)$ for which $p(\cdot, \mu)$ $\in C(V)$. A charge $\mu \in a(V, Y)$ is said to be Rickart on the ring $V$ if $\lim _{n} \mu\left(A_{n}\right)=0$ for each dominated, disjoint sequence $A_{n} \in V, n \in N$. Let $R(V, Y)$ denote the family of Rickart vector charges on $V$. It is known that $R(V, Y) \subseteq a b(V, Y)$ and that $R(V, Y)=a b(V, Y)$ if the space $Y$ does not contain a copy of the space $c_{0}$ of null convergent sequences of scalars.

In this paper it is shown that each uniformly Rickart, pointwise bounded family of additive contents on a ring of sets admitting an upper complete, additive content generates an additive control content. The known control measure existence theorems (see for example [2], [3] and [5]) are included in this formulation. The control measure existence theorem is then specialized to obtain a not necessarily additive control content for an arbitrary uniformly Rickart family of contents containing an upper complete content.

The vector form of Phillips' lemma [10] established by Brooks and Jewett [4] is used to show that each sequence of finitely additive, Rickart vector charges, converging pointwise on a delta ring of sets, is uniformly Rickart. 
This result is combined with the control charge existence theorems to establish a Vitali-Hahn-Saks theorem which contains the Bogdanowicz version [8], obtained independently by Labuda [6], and the Brooks-Jewett extension [4].

A content $q \in C(V)$ is said to be monotonically Cauchy if every monotone, dominated sequence from the ring $V$ is $q$-Cauchy. A family of contents $Q \subset C(V)$ is said to be uniformly, monotonically Cauchy if every monotone, dominated sequence from the ring $V$ is $q$-Cauchy uniformly with respect to the contents $q \in Q$.

A content $q \in C(V)$ is said to be Ando if for every number $\varepsilon>0$ and every dominated (not necessarily disjoint) sequence $A_{n} \in V, n \in N$, there exists a strictly increasing map $j: N \rightarrow N$ such that the sequences of sets $B_{n}, C_{n} \in V, n \in N$, defined by the relations

$$
B_{n}=A_{n} \cup A_{n+1} \cup \cdots \cup A_{j(n)} \text { and } C_{n}=\bigcap_{k=1}^{n} B_{k} \text {, }
$$

satisfy $q\left(B_{n} \backslash C_{n}\right)<\varepsilon$ for all indices $n \in N$. A family of contents $Q \subset C(V)$ is said to be uniformly Ando if the above inequality holds uniformly with respect to the contents $q \in Q$. This definition is motivated by a result of Ando [1, pp. 396-397], who proved that this condition is satisfied for the pointwise supremum of a uniformly monotonically Cauchy family of real, finitely additive measures on a $\sigma$-algebra of sets.

Let $Q \subset C(V)$ be a uniformly Rickart family of contents and let $\varphi$ be a subadditive, strictly increasing, nonnegative continuous function on the nonnegative reals $R^{+}$which vanishes at zero. Then the family $\varphi \circ Q$ is uniformly Rickart on the ring $V$ and equivalent to the family $Q$. Moreover, if the function $\varphi$ is also bounded, then the family $\varphi \circ Q$ is equicontinuous with respect to the content $p(\cdot)=\sup (\varphi \circ q(\cdot): q \in Q)$. This observation yields the following characterization of uniformly Rickart families of contents by permitting a reduction of the theorem to an equivalent statement for a single content - where the characterization is clear.

LEMMA 1. Let $V$ be a ring of subsets of an abstract space $X$. For a family $Q \subset C(V)$, the following conditions are equivalent.

(1) The family $Q$ is uniformly Rickart on the ring $V$.

(2) The family $Q$ is uniformly monotonically Cauchy on the ring $V$.

(3) The family $Q$ is uniformly Ando on the ring $V$.

(4) For each dominated, disjoint sequence $A_{n} \in V, n \in N$, and each number $\varepsilon>0$ there exists a finite set $\Delta \subset N$ such that $q\left(\cup_{k \in \Delta^{\prime}} A_{k}\right)<\varepsilon$ for all finite sets $\Delta^{\prime} \subset N$ for which $\Delta \cap \Delta^{\prime}=\varnothing$ and for all contents $q \in Q$.

THEOREM 1. Let $V$ be $a$ ring of subsets of an abstract space $X$ and assume that the ring $V$ admits an upper complete, additive content $q_{0} \in C(V)$. Let $Q \subset C(V)$ have the following properties:

(1) The family $Q$ is uniformly Rickart on the ring $V$.

(2) Each member of the family $Q$ is additive.

(3) For each set $A \in V, m(A)=\sup (q(A), q \in Q)<\infty$. 
Then there exists an additive (and hence Rickart) content $p \in C(V)$ such that the family $Q$ is p-equicontinuous.

Proof. Let $Q_{0}=Q \cup\left\{q_{0}\right\}$. Then the family of contents $Q_{0} \subset C(V)$ satisfies conditions (1), (2) and (3) of the theorem. The assertion is established by showing first that for each number $\varepsilon>0$, there exists a number $\delta>0$ and a finite family $q_{1}, \ldots, q_{n} \in Q_{0}$ such that $A \in V$ and $q_{k}(A)<\delta$ for $k=$ $1,2,3, \ldots, n$, yields $q(A)<\varepsilon$ for all contents $q \in Q_{0}$. If that is not the case, then there exists a number $\varepsilon>0$ with the following property: for each number $\delta>0$ and each finite set $q_{1}, \ldots, q_{n} \in Q_{0}$, there exists a set $A \in V$ and a content $q \in Q_{0}$ such that $q_{t}(A)<\delta$ for $t=1,2,3, \ldots, n$ and $q(A) \geqslant$ $\varepsilon$. Beginning with the upper complete content $q_{0} \in Q_{0}$ and the number $\delta_{0}=1$, we may apply the hypothesis inductively to choose a sequence of sets $A_{n} \in V$, and a sequence of .contents $q_{n} \in Q_{0}$, such that $q_{t}\left(A_{n}\right)<2^{-n}$ for $t=0,1,2, \ldots, n$ and $q_{n+1}\left(A_{n}\right) \geqslant \varepsilon$. Notice that the sequence $A_{n} \in V, n=$ $0,1,2, \ldots$, is summable with respect to the upper complete content $q_{0} \in$ $Q_{0}$. Thus, the sequence $A_{n} \in V, n=0,1,2, \ldots$, is dominated. It follows from the lemma that there exists a strictly increasing map $j: n \rightarrow j(n)$ such that $q\left(B_{n} \backslash C_{n}\right)<\varepsilon$, for all $n \in N$ and for all contents $q \in Q_{0}$, where $B_{n}=$ $A_{n} \cup A_{n+1} \cup \cdots \cup A_{j(n)}$ and $C_{n}=\bigcap_{k=1}^{n} B_{k}$. From the construction, for integers $k, n$ with $k \leqslant n$,

$$
q_{k}\left(B_{n}\right) \leqslant \sum_{t=n}^{j(n)} q_{k}\left(A_{t}\right)<\sum_{t=n}^{\infty} 2^{-t}=2^{-(n-1)} .
$$

This yields, for $k=0,1,2, \ldots, \lim _{n} q_{k}\left(B_{n}\right)=0$. The monotonicity of each content $q_{k}: k=0,1,2, \ldots$ yields $\lim _{n} q_{k}\left(C_{n}\right)=0$ for each $k=0,1,2, \ldots$ Since the set $Q_{0}$ is uniformly Rickart on the ring $V$, this limit is uniform in the index $k=0,1,2, \ldots$ (that is, $\lim _{n} q_{k}\left(C_{n}\right)=0$ uniformly for $k=$ $0,1,2, \ldots)$. Thus, there exists a positive integer $n(\varepsilon)$ such that $n>n(\varepsilon)$ yields $q_{k}\left(C_{n}\right)<\varepsilon / 2$ for all $k=0,1,2, \ldots$ The inequalities

$$
q_{k}\left(A_{n}\right) \leqslant q_{k}\left(B_{n}\right) \leqslant q_{k}\left(C_{n}\right)+q_{k}\left(B_{n} \backslash C_{n}\right)
$$

insure that $q_{k}\left(A_{n}\right)<\varepsilon$ for all $n \geqslant n(\varepsilon)$ and all $k=0,1,2, \ldots$ However, this clearly contradicts the relation $q_{n+1}\left(A_{n}\right) \geqslant \varepsilon$ for $n=0,1,2, \ldots$

We have thus established that the set $Q$ is equicontinuous with respect to the topological ring $\left(V, Q_{0}\right)$. Now, for each index $n \in N$ use the established equicontinuity to choose a finite set $\left\{q_{n 1}, q_{n 2}, \ldots, q_{n k_{n}}\right\} \subset Q_{0}$ and a number $\delta_{n}>0$ such that $A \in V$ and $q_{n j}(A)<\delta_{n}$ for $j=1, \ldots, k_{n}$ yields $q(A)<2^{-n}$ for all contents $q \in Q$. Define for $A \in V$,

$$
p(A)=\sum_{n=1}^{\infty} 2^{-n} \cdot \sum_{j=1}^{k_{n}} \frac{q_{n j}(A)}{k_{n}} .
$$

Condition (3) insures that $p$ is a well-defined, additive content. Moreover, since the set of contents $Q_{0} \subset C(V)$ is uniformly Rickart, the content $p$ is Rickart. 
To see that the set $Q$ is equicontinuous with respect to additive content $p$, consider any set $A \in V$ such that $p(A)<\delta_{n} 2^{-n} k_{n}^{-1}$. Then for $j=$ $1,2,3, \ldots, k_{n}, q_{n j}(A)<\delta_{n}$. From the construction of the set $\left\{q_{n j}: j=\right.$ $\left.1,2, \ldots, k_{n}, n=1,2, \ldots\right\} \subset Q_{0}$, we conclude $q(A)<1 / 2^{n}$ uniformly with respect to the contents $q \in Q_{0}$. The inclusion $Q \subset Q_{0}$ then insures that the set $Q$ is $p$-equicontinuous.

When the ring $V$ is an algebra or a $\sigma$-ring of subsets of the space $X$, every content is upper complete. In either of these situations, the initial assumption of Theorem 1 is always satisfied. In the literature, these are precisely the two cases for which a control measure is known to exist, e.g., Bartle, Dunford and Schwartz [2] or Brooks [3]. Since each uniformly strongly bounded family of additive contents on a ring admits an extension to a uniformly strongly bounded family of additive contents on the generated algebra, the Drewnowski [5] control charge existence theorem is also contained in Theorem 1.

If $V$ is the family of summable sets for some scalar measure, none of the standard control content existence theorems apply; however, Theorem 1 may be invoked to insure existence. General necessary and sufficient conditions to insure the existence of an upper complete, additive content on an arbitrary ring are not known.

Theorem 1 has a slightly stronger formulation that is useful in extension theory. The stronger formulation may be obtained without change in the method of proof but does require refined definitions of the Rickart and upper completeness conditions and the observation that the lemma may be reformulated so as to remain valid for the refined definitions.

Let $V_{\sigma}$ denote the family of all sets expressible as a countable union of sets from the ring $V$ and let $W \subset V_{\sigma}$ be an arbitrary family of sets. A content $q \in C(V)$ is said to be Rickart on the ring $V$ relative to $W$ if $\lim _{n} q\left(A_{n}\right)=0$ for each disjoint, $W$-dominated sequence $A_{n} \in V, n \in N$. A family of contents $Q \subset C(V)$ is said to be uniformly Rickart on the ring $V$ relative to $W$ if the above limit holds uniformly with respect to $q \in Q$. It should be noted that any additive content $q \in C(V)$ is Rickart on the ring $V$ relative to the family $W=\left\{A \in V_{\sigma}: \sup (q(B): B \in V, B \subseteq A)<\infty\right\}$. A content $q \in$ $C(V)$ is said to be upper complete on $V$ relative to $W$ if for each sequence $A_{n} \in V, n \in N$ for which $\Sigma_{n} q\left(A_{n}\right)<\infty$, there exists a set $B \in W$ with $\cup_{n} A_{n} \subseteq B$. That is, a content $q \in C(V)$ is upper complete on $V$ relative to $W$ if the only $q$-summable sequences are $W$-dominated.

Corollary. Let $v \in C(V)$ be an additive (countably additive) content that is upper complete and Rickart on $V$ relative to a family $W \subset V_{\sigma}$. Let $Q \subset$ $C(V)$ have the following properties:

(1) The family $Q$ is uniformly Rickart on the ring $V$ relative to the family $W$.

(2) Each member of the set $Q$ is additive (countably additive) on the ring $V$.

(3) For each set $A \in V$, $\sup (q(A): q \in Q)<\infty$.

Then there exists an additive (countably additive) content $w \in C(V)$ such that the family $Q$ is w-equicontinuous. 
As an illustration of the corollary, let $v \in C(V)$ be countably additive. Then it is both Rickart and upper complete on $V$ relative to the family $W=\left\{A \in V_{\sigma}: \sup (v(B): B \in V, B \subseteq A)<\infty\right\}$. Then any family of countably additive contents $Q$ satisfying conditions (1), (2) and (3) of the corollary admits a countably additive control content $w \in C(V)$ that is Rickart on $V$ relative to $W$. But the content $w$, being countably additive, admits an extension (denoted $\tilde{w}$ ) defined on the delta ring $\tilde{W}$ of $\tilde{w}$-summable sets. That is, $\tilde{W}$ is nothing but the $w^{*}$-measurable sets with the finite outer measure. Moreover, the ring $V$ is dense in the topological ring $(\tilde{W}, \tilde{w})$. Consequently, the members of the set $Q$ are simultaneously extendible to the delta ring $\tilde{W}$. Applications of this result to the theory of vector measures are developed in [9].

A proof essentially identical to the proof of Theorem 1 establishes the validity of the following variation of the control content existence theorem.

THEOREM 2. Let $V$ be a ring of the subsets of an abstract space $X$ and assume that a family of contents $Q \subset C(V)$ contains an upper complete content. Then the set $Q$ is uniformly Rickart on the ring $V$ if and only if there exists a Rickart content $p \in C(V)$ such that:

(1) The set $Q$ is p-equicontinuous.

(2) The topological rings $(V, Q)$ and $(V, p)$ are equivalent.

It should be noted that the equivalent content is, in general, not equal to the pointwise supremum of the underlying family of contents.

The vector formulation of the Vitali-Hahn-Saks theorem requires a discussion of the properties of pointwise convergent sequences of Rickart vector charges as developed by Brooks and Jewett [4]. The fundamental result is the Banach space formulation of Phillips' lemma [10], which Brooks and Jewett [4] have established.

Let $P(N)$ denote all subsets of the natural numbers $N$. For each vector charge $\mu \in R(P(N), Y)$ define a charge $\mu^{*} \in c a(P(N), Y)$ by the relation $\mu^{*}(\Delta)=\sum_{k \in \Delta} \mu(\{k\})$ for each set $\Delta \in P(N)$. Since the charge $\mu$ is Rickart, the series $\sum_{k=1}^{\infty} \mu(\{k\})$ is unconditionally convergent. Therefore, the mapping $\mu^{*}: \Delta \rightarrow \mu^{*}(\Delta), \Delta \in P(N)$, is well defined. The countable additivity is an immediate consequence of the definition. The charges $\mu$ and $\mu^{*}$ agree on finite sets of positive integers. The vector charge $\mu^{*}$ may be considered as the countably additive part of the vector charge $\mu$.

Theorem 3 (Phillips, Brooks, Jewett). Let the sequence $\mu_{n} \in$ $R(P(N), Y), n \in N$, be pointwise convergent to zero (i.e., $\lim _{n} \mu_{n}(\Delta)=0$ for each $\Delta \in P(N)$ ). Then $\lim _{n} \mu_{n}^{*}(\Delta)=0$ uniformly with respect to the sets $\Delta \in$ $P(N)$.

The following proposition has been established by Brooks and Jewett [4] for sequences of finitely additive, strongly bounded vector measures on a $\sigma$-algebra. The Brooks-Jewett arguments carry over to sequences of Rickart 
vector charges converging pointwise on a delta ring.

THEOREM 4. Let $V$ be a delta ring (i.e. a ring closed under countable intersections) of subsets of the space $X$ and let $\mu_{n} \in R(V, Y), n \in N$, be pointwise convergent on the delta ring $V$. If for each set $A \in V, \mu(A)=$ $\lim _{n} \mu_{n}(A)$, then $\mu \in R(V, Y)$.

The following theorem is an immediate consequence of Theorem 4.

THEOREM 5. Let $V$ be a delta ring of subsets of the space $X$ and let $\mu_{n} \in R(V, Y), n \in N$, be pointwise convergent on $V$. Then the sequence $\mu_{n}, n$ $\in N$, is uniformly Rickart.

Brooks and Jewett [4] established that pointwise convergent sequences of Rickart vector charges on a $\sigma$-algebra of sets are uniformly additive (see [4] for the definition). Ando [1] showed that pointwise converging sequences of scalar charges on a $\sigma$-algebra of sets are uniformly Cauchy with respect to dominated monotone convergence. In fact, Ando's argument, combined with the vector form of Phillips' lemma, may be used to establish that sequences of vector charges which converge pointwise on a delta ring of sets are uniformly Cauchy (definition analogous to that for contents) with respect to dominated, monotone convergence. The equivalence of the above two conditions to the uniform Rickart condition is established in [8].

Theorem 3 and the following lemma provide the essential steps in establishing a general Vitali-Hahn-Saks theorem (Saks [12] and [13], Nikodým, [7]).

LeMma 2. Let $p \in C(V)$ be upper complete and let $Q \subset C(V)$ have the following properties:

(1) The family $Q$ is uniformly Rickart on $V$.

(2) Each content $q \in Q$ is p-continuous.

Then the family $Q$ is p-equicontinuous.

Proof. Note first that for each decreasing sequence $C_{n} \in V, n \in N$, for which $\lim _{n} p\left(C_{n}\right)=0$, one has $\lim _{n} q\left(C_{n}\right)=0$ uniformly with respect to the contents $q \in Q$. Indeed, property (1) insures that the limits $r(q)=\lim _{n} q\left(C_{n}\right)$ exist uniformly with respect to $q \in Q$ and from property (2), $r(q)=0$ for each $q \in Q$. The lemma may now be established by assuming the contrary and proceeding just as in Theorem 1 with the Ando relations.

For any topological ring of sets $(V, P)$, let $K\left((V, P), R^{+}\right)$denote the space of $P$-continuous contents on the ring $V$ and denote by $a b c((V, P), Y)$ the space of locally bounded, $P$-continuous vector charges. The definition of the semivariation insures that for each charge $\mu \in a b c((V, P), Y), p(\cdot, \mu) \in$ $K\left((V, P): R^{+}\right)$.

Theorem 6 (Vitali-Hahn-SAKs). Let $V$ be a delta ring of subsets of the space $X$ and let $p \in C(V)$ be an upper complete content. If a sequence $\mu_{n} \in a b c((V, p), Y) \cap R(V, Y), n \in N$, converges pointwise on the delta ring 
$V$, then the sequence $\mu_{n}, n \in N$, is p-equicontinuous.

Proof. Since the ring $V$ is assumed to be a delta ring, Theorem 5 insures that the sequence $\mu_{n}, n \in N$, is uniformly Rickart. Therefore, the set of contents $\left\{p\left(\cdot, \mu_{n}\right): n=1,2,3, \ldots\right\}$ is uniformly Rickart on $V$. Since the content $p \in C(V)$ is upper complete and $\left\{p\left(\cdot, \mu_{n}\right): n=1,2,3, \ldots\right\} \subset$ $K\left((V, p): R^{+}\right)$, Lemma 2 insures that the set $\left\{p\left(\cdot, \mu_{n}\right), n=1,2,3, \ldots\right\}$ is $p$-equicontinuous. The relation $\left|\mu_{n}(\cdot)\right| \leqslant p\left(\cdot, \mu_{n}\right)$ for $n=1,2,3, \ldots$ insures that the sequence $\mu_{n}, n \in N$, is $p$-equicontinuous.

As a corollary, one obtains a particular case of the Bogdanowicz [8] and Drewnowski [5] and Labuda [6] formulation of the Vitali-Hahn-Saks theorem. This formulation was first established by a Baire category argument combined with the observation that a topological ring $(V, p)$ is sequentially complete if and only if $V$ is a delta ring, and the content $p$ is Daniell continuous and upper complete. Such a topological ring is referred to as a dominated and absolute convergence ring of sets. The name is motivated by some equivalent formulations of the completeness condition.

COROllary. Let $(V, p)$ be a dominated and absolute convergence ring of sets. Let $\mu_{n} \in a(V, Y), n \in N$, be $p$-continuous and converge pointwise on the ring $V$. Then the sequence $\mu_{n}, n \in N$, is p-equicontinuous.

A set $K \subset a(V, Y)$ is said to be sequentially $V$-pointwise compact if every infinite sequence in the set $K$ contains a subsequence which converges pointwise on the ring. Theorems 1,4 and 5 yield the following equicontinuity condition for sequential $V$-pointwise compactness for delta rings admitting an upper complete additive content.

THEOREM 7. Let $V$ be a delta ring of subsets of an abstract space $X$ and assume that the ring $V$ admits an upper complete, additive content. Let $K \subset$ $R(V, Y)$ be sequentially $V$-pointwise compact in the space $a(V, Y)$. Then there exists an additive content $p \in C(V)$ such that the set $K$ is p-equicontinuous.

COROllary 1. Let $V$ be a delta ring of subsets of an abstract space $X$ and assume that the ring $V$ admits an upper complete additive content. Let $\mu_{n} \in$ $R(V, Y), n \in N$, be pointwise convergent on $V$. Then there exists an additive content $p \in C(V)$ such that the sequence $\mu_{n}, n \in N$, is p-equicontinuous.

Corollary 2. Let $V$ be a o-ring of subsets of the space $X$ and let $K \subset R(V, Y)$ be sequentially $V$-pointwise compact in the space $a(V, Y)$. Then there exists an additive content $p \in C(V)$ such that the set $K$ is p-equicontinuous and the topological rings $(V, p)$ and $(V, p(\cdot, \mu): \mu \in K)$ are equivalent.

\section{REFERENCES}

1. T. Ando, Convergent sequences of finitely additive measures, Pacific J. Math. 11 (1960), 395-404. MR 25 \# 1255.

2. R. G. Bartle, N. Dunford and J. Schwartz, Weak compactness and vector measures, Canad. J. Math. 7 (1955), 289-305. MR16, 1123. 
3. J. K. Brooks, On the existence of a control measure for strongly bounded vector measures, Notices Amer. Math. Soc. 18 (1971), 415. Abstract \#71T-B58.

4. J. K. Brooks and R. S. Jewett, On finitely additive vector measures, Proc. Nat. Acad. Sci. U.S.A. 67 (1970), 1294-1298. MR 42 \#4697.

5. L. Drewnowski, Topological rings of sets, continuous set functions, integration. I, II, III, Bull. Acad. Polon. Sci. Sér. Sci. Math. Astronom. Phys. 20 (1972), 269-276, 277-286; ibid. 20 (1972), 439-445. MR 46 \# 5558; 47 \#5200.

6. I. Labuda, Sur quelques généralisations des théorèmes de Nikodým et de Vitali-Hahn-Saks, Bull. Acad. Polon. Sci. 20 (1972), 447-456. MR 49 \#5290.

7. $\mathrm{O}$. Nikodym, Sur les suites convergentes de fonctions parfaitemente additives d'ensemble abstract, Monatsh. Math. Phys. 40 (1933), 427-432.

8. R. A. Oberle, Theory of a class of vector measures on topological rings of sets and generalizations of the Vitali-Hahn-Saks theorem, Catholic Univ., 1971.

9. $\quad$ A note on equicontinuous families of volumes with an application to vector measures, Canad. J. Math. 26 (1974), 273-280. MR 49 \# 524.

10. R. S. Phillips, On linear transformations, Trans. Amer. Math. Soc. 48 (1940), 516-541. MR 2, 318.

11. C. E. Rickart, Decompositions of additive set functions, Duke Math. J. 10 (1943), 653-665. MR 5, 232.

12. S. Saks, On some functionals. I, Trans. Amer. Math. Soc. 35 (1933), 549-556.

13. __ Additions to the note on some functionals, Trans. Amer. Math. Soc. 35 (1933), 965-970.

The Center for Naval Analyses, Arlington, Virginia 22209 